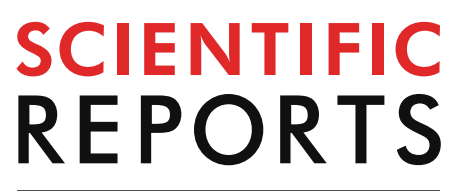

natureresearch

Check for updates

\title{
Development of fully automated and ultrasensitive assays for urinary adiponectin and their application as novel biomarkers for diabetic kidney disease
}

Toshihiro Watanabe ${ }^{1 凶}$, Yuki Fujimoto ${ }^{2}$, Aya Morimoto $^{3}$, Mai Nishiyama ${ }^{3}$, Akinori Kawai $^{3}$, Seiki Okada ${ }^{3}$, Motohiro Aiba ${ }^{2}$, Tomoharu Kawano ${ }^{4}$, Mina Kawahigashi ${ }^{4}$, Masashi Ishizu ${ }^{5}$, Hiroyasu Mori ${ }^{5}$, Munehide Matsuhisa ${ }^{5}$, Akiko Hata ${ }^{6}$, Makoto Funaki ${ }^{6}$ \& Seiichi Hashida

Glomerular filtration rate (GFR) and urinary albumin excretion rate (UAER) are used to diagnose and classify the severity of chronic kidney disease. Total adiponectin (T-AN) and high molecular weight adiponectin (H-AN) assays were developed using the fully automated immunoassay system, $\mathrm{HI}-1000$ and their significance over conventional biomarkers were investigated. The T-AN and H-AN assays had high reproducibility, good linearity, and sufficient sensitivity to detect trace amounts of adiponectin in the urine. Urine samples after gel filtration were analyzed for the presence of different molecular isoforms. Low molecular weight (LMW) forms and monomers were the major components (93\%) of adiponectin in the urine from a diabetic patient with normoalbuminuria. Urine from a microalbuminuria patient contained both high molecular weight (HMW) (11\%) and middle molecular weight (MMW) (28\%) adiponectin, although the LMW level was still high (52\%). The amount of HMW (32\%) and MMW (42\%) were more abundant than that of LMW (24\%) in a diabetic patient with macroalbuminuria. T-AN $(r=-0.43)$ and $\mathrm{H}-\mathrm{AN}(\mathrm{r}=-0.38)$ levels showed higher correlation with estimated GFR (eGFR) than UAER $(r=-0.23)$. Urinary levels of both T-AN and H-AN negatively correlated with renal function in diabetic patients and they may serve as new biomarkers for diabetic kidney disease.

Chronic kidney disease (CKD) is a disease, in which chronic renal impairment or decline in renal function is followed by an end-stage renal disease that requires dialysis or a kidney transplant ${ }^{1}$. In addition, CKD has been shown to increase the risk of cardiovascular disease (CVD) such as myocardial infarction, stroke, and heart failure, as well as death ${ }^{2}$. Therefore, early diagnosis and appropriate treatment of CKD is critical to prevent the onset/progression of CKD and the development of CVD. Glomerular filtration rate (GFR) and urinary albumin excretion rate (UAER) have been used to diagnose and classify the severity of $\mathrm{CKD}^{3}$, and previous studies have shown that urinary adiponectin may be useful as a biomarker of CKD, as well.

Several studies have confirmed that urinary adiponectin increases with the progression of renal impairment in patients with diabetes ${ }^{4-6}$. It has also been reported that predicting the onset/progression of such impairments by either GFR or UAER is difficult, but urinary adiponectin levels may enable this prediction ${ }^{7,8}$. Furthermore, increased urinary adiponectin has been reported in $\operatorname{IgA}$ nephropathy ${ }^{9}$ and in nephropathy caused by systemic lupus erythematosus ${ }^{10,11}$. Urinary adiponectin has been thought to be excreted due to either disruption of the

${ }^{1}$ R\&D Division, Sysmex R\&D Center Americas, Inc., Mundelein, IL, USA. ${ }^{2}$ Life Style Diseases, Institute for Health Sciences, Tokushima Bunri University, Tokushima, Japan. ${ }^{3}$ Clinical Innovation, Sysmex Corporation, Kobe, Hyogo, Japan. ${ }^{4}$ Human Life Science, Tokushima Bunri University, Tokushima, Japan. ${ }^{5}$ Diabetes Therapeutics and Research Center, Institute of Advanced Medical Sciences, Tokushima University, Tokushima, Japan. ${ }^{6} \mathrm{Clinical}$ Research Center for Diabetes, Tokushima University Hospital, Tokushima, Japan. ${ }^{7}$ Department of Diabetes and Molecular Genetics, Ehime University Graduate School of Medicine, Ehime, Japan. ${ }^{\circledR}$ email: Watanabe.Toshihiro@sysmex.co.jp 


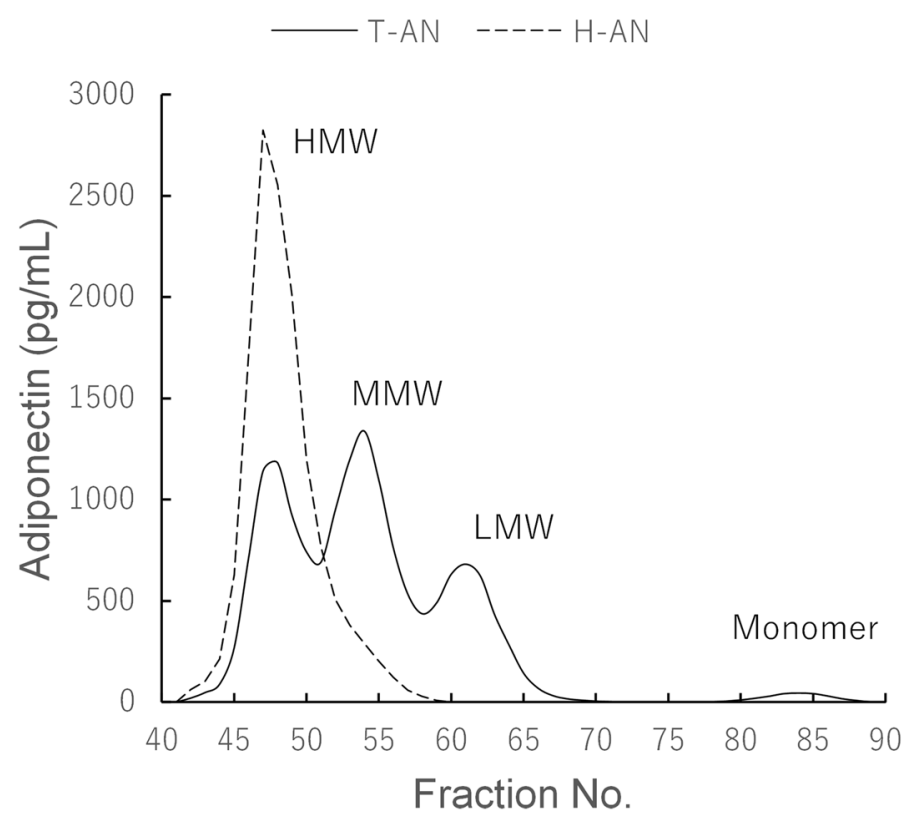

Figure 1. Reactivities of T-AN and $\mathrm{H}-\mathrm{AN}$ assays to multimers and monomer fractions of urinary adiponectin. SEC fractions of a urine sample from a patient with macroalbuminuria were analyzed with T-AN and H-AN assays.

glomerular barrier ${ }^{8-10}$, tubular injuries ${ }^{8,12}$ or vascular damages ${ }^{13}$, and it is of interest to investigate if urinary adiponectin would have significance as a biomarker over other conventional biomarkers in diabetic kidney disease (DKD).

Immunoassay technology is widely used for detection of protein biomarkers and novel technologies are continuing to be developed ${ }^{14-21}$. Adiponectin is present in urine at very low concentrations (pg/mL levels) $)^{4-13}$ and a commercial immunoassay kit do not have sufficient analytical sensitivity to detect these trace amounts ${ }^{10}$. In recent years, an ultrasensitive immunoassay system capable of measuring down to a concentration of pg/ $\mathrm{mL}$ or less has been put into practical use ${ }^{22,23}$. Immune complex transfer enzyme immunoassay (ICT-EIA) ${ }^{24}$, which is one of the methods to realize ultrasensitive immunoassay detection, can be adapted to high-throughput immunoassay systems used in clinical settings ${ }^{25-28}$. Using antibody-coated magnetic particles to increase capture efficiency, immune complex transfer enables interfering background signal to be eliminated, thereby enhancing analytical sensitivity.

Previously, we developed a manual ICT-EIA to detect trace amounts of total adiponectin (T-AN) in urine e. $^{29}$. We have also reported that patients with diabetes show increased T-AN levels in their urine, in spite of a decrease in their blood, when compared with healthy and obese subjects. Analysis of urine by gel filtration revealed that urine T-AN consists of four isoforms, which are high molecular weight (HMW), medium molecular weight (MMW), low molecular weight (LMW), and monomers. HMW adiponectin (H-AN) is increased in the urine of a patient with microalbuminuria compared with that in the urine of a patient with normoalbuminuria. Furthermore, T-AN concentrations, but not UAER, shows a significant correlation with eGFR. As a result, urinary adiponectin may be a useful surrogate marker for the decline of eGFR.

As described above, the molecular morphology of urinary adiponectin may be altered in response to the progression of DKD. Therefore, we developed a method, which is capable of differentially quantifying all adiponectin isoforms, which is sensitive enough to detect trace amounts of them found in the urine. T-AN assays, which we reported previously ${ }^{29}$, was performed manually with multiple steps. Thus, it is complicated and time consuming, and it may be challenging to conduct in clinical situations. In this paper, we demonstrate our fully automated ultrasensitive T-AN assay and a new H-AN assay, which is also ultrasensitive and fully automated. Furthermore, we show the significance of these two assays as new biomarkers for DKD.

\section{Results}

Reactivities of T-AN and H-AN assays to adiponectin multimers and monomer fractions. A urine sample obtained from a patient with macroalbuminuria was fractionated using size exclusion chromatography (SEC) and fractions were analyzed using both T-AN and H-AN assays (Fig. 1). Similar to our previous study, in which T-AN assays were conducted manually ${ }^{29}, 4$ peaks, which correspond to HMW, MMW, LMW and monomeric adiponectin, respectively, were observed by the T-AN assay. In contrast, the H-AN assay generated a single peak that corresponded to HMW adiponectin, although a weak signal without a peak was also detected in fractions corresponding to MMW adiponectin. However, the MMW signal is remarkably low compared to the signal corresponding to HMW, demonstrating the specificity of our H-AN assay for reporting the amount of HMW in the urine. 
a

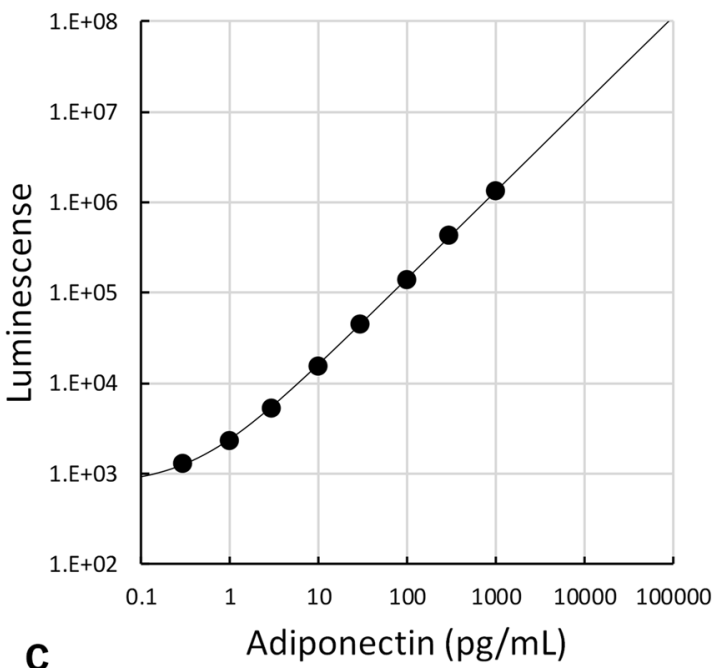

C

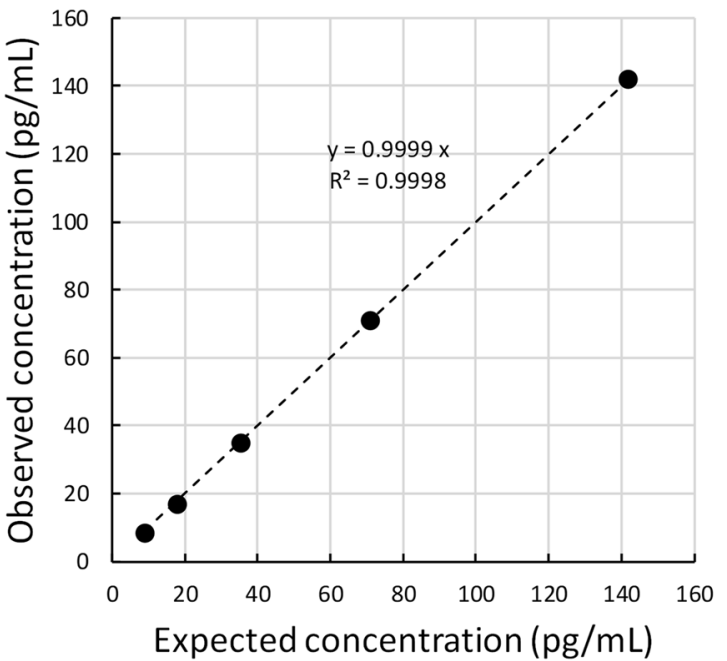

b
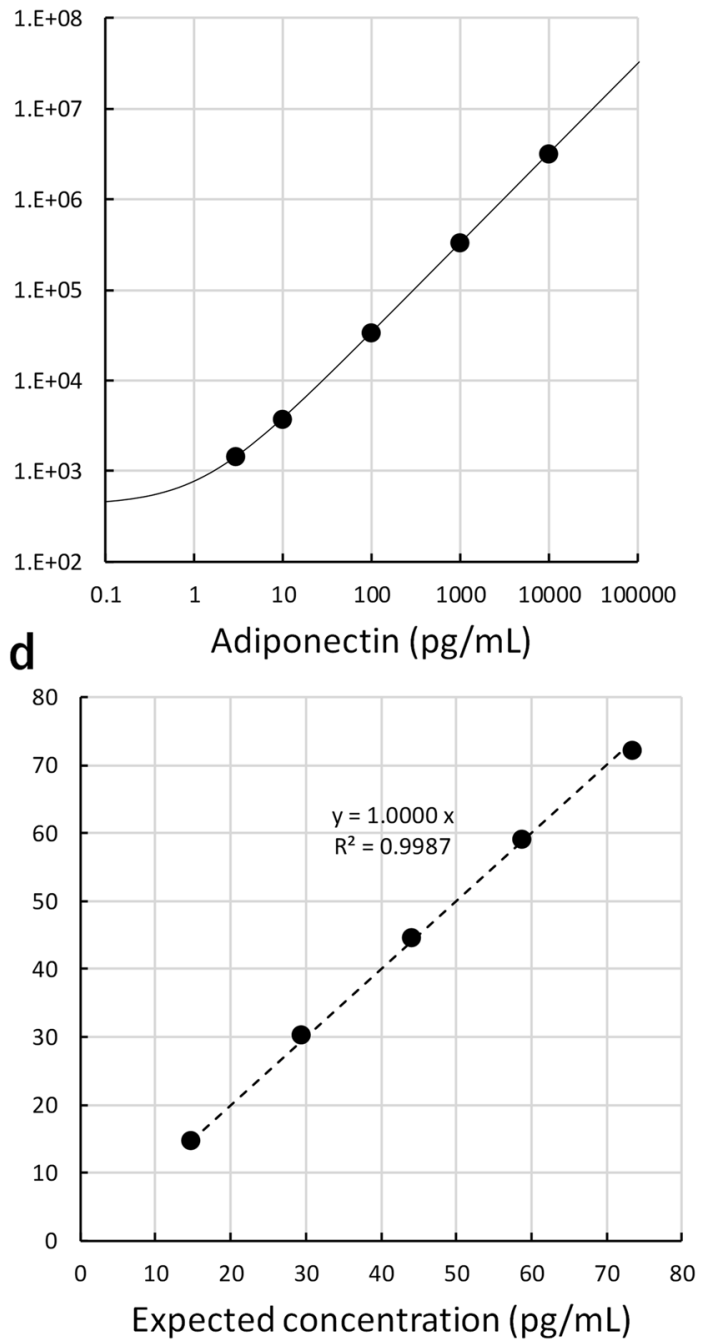

Figure 2. Analytical performance of T-AN and H-AN assays. Dose and response curves for T-AN (a) and $\mathrm{H}-\mathrm{AN}(\mathbf{b})$ assays. Dilution linearity of T-AN (c) and H-AN (d) assays.

Analytical performance of T-AN and H-AN assays. Analytical performance of T-AN and H-AN assays are shown in Fig. 2. Dose and response curves were obtained for these two assays (Fig. 2a,b) and LoDs (Limits of Detection) were calculated as 0.05 and $0.11 \mathrm{pg} / \mathrm{mL}$ for T-AN and H-AN assays, respectively, using the mean plus 3SD of low concentration samples. Reproducibility was good with CVs of $1.8-2.6 \%$ and $2.1-5.1 \%$ $(\mathrm{n}=10)$ for T-AN and H-AN assays, respectively. In testing of serially diluted samples (Fig. 2c,d), the ratios of the observed concentrations to the expected concentrations based on the dilution factor in the T-AN and H-AN assays were $93-100 \%$ and $98-103 \%$, respectively.

A manual ICT-EIA for T-AN was previously developed and a feasibility study of urinary T-AN levels as a CKD biomarker was reported ${ }^{29}$. The newly developed T-AN assay uses the same antibody pair, and has a comparable sensitivity with the manual ICT-EIA, while the assay time was shortened from 3 days to $1 \mathrm{~h}$ because of the fully automated process on HI-1000. A measurement by the manual ICT-EIA and a measurement by the T-AN assay on HI-1000 showed a strong correlation (Fig. 3). However, for samples containing high levels of T-AN (above $50 \mu \mathrm{g} / \mathrm{gCr}$ ), the measurement on HI-1000 tended to be lower than that by the manual ICT-EIA (Fig. 3a). When those samples were excluded, the slope was close to 1 (Fig. 3b).

Composition of adiponectin multimers in the urine of DKD patients. In addition to the data of a patient with macroalbuminuria shown in Fig. 1, urine samples from a patient with normoalbuminuria and a patient with microalbuminuria were also fractionated by SEC and assayed for T-AN, H-AN, albumin and creatinine (Fig. 4) and the background data for these 3 cases are shown in Table 1. Both T-AN and H-AN levels were higher in the urine of patients with either microalbuminuria or macroalbuminuria, which could be mainly attributed to the appearance of HMW and MMW in the urine of those patients. 


\section{a}

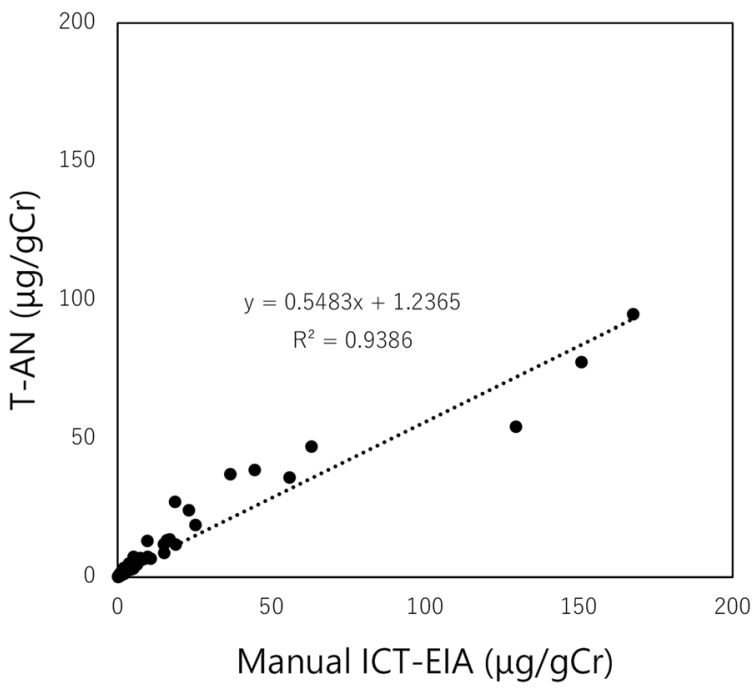

b

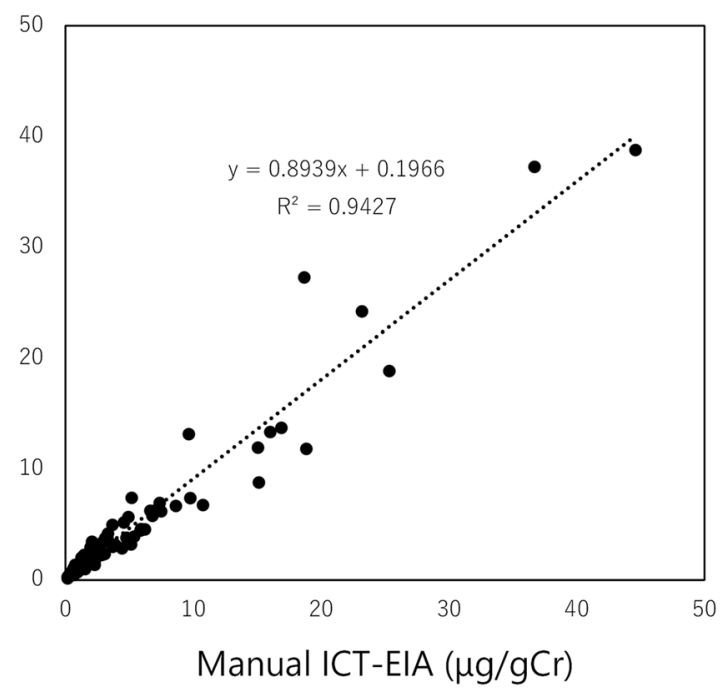

Figure 3. Correlation between two assays for T-AN levels in the urine; a manual assay (Manual ICT-EIA) and a fully automated T-AN assay on HI-1000 (T-AN.) (a) All samples, (b)samples with a measurement of T-AN levels by the manual ICT-EIA $<50 \mu \mathrm{g} / \mathrm{gCr}$.
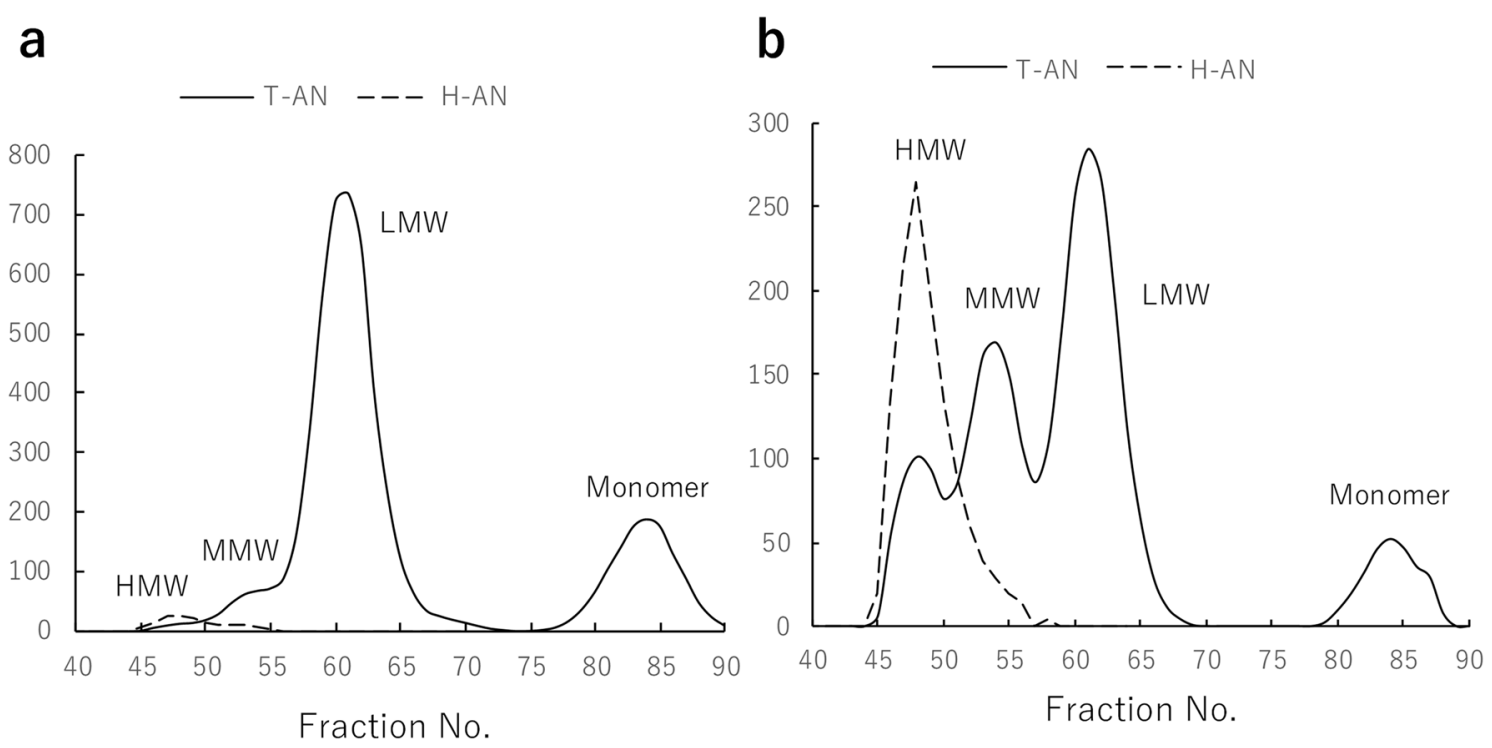

Figure 4. Composition of adiponectin in urine from patients with normoalbuminuria (a) and microalbuminuria (b). Urine samples from a patient with normoalbuminuria and a patient with microalbuminuria were separated by SEC and fractions were measured with T-AN and H-AN assays.

\begin{tabular}{|l|l|l|l|}
\hline \multirow{2}{*}{} & Albuminuria & Micro (Fig. 4b) & Macro (Fig. 1) \\
\cline { 2 - 4 } & Normo (Fig. 4a) & 83 & 2926 \\
\hline Albumin $(\mathrm{mg} / \mathrm{gCr})$ & 7.5 & 19 & 78 \\
\hline T-AN $(\mu \mathrm{g} / \mathrm{gCr})$ & 5.7 & 8.6 & 78 \\
\hline H-AN $(\mu \mathrm{g} / \mathrm{gCr})$ & 0.14 & 34 & 43 \\
\hline eGFR $\left(\mathrm{mL} / \mathrm{min} / 1.73 \mathrm{~m}^{2}\right)$ & 100 & & 43 \\
\hline
\end{tabular}

Table 1. Urinary biomarker concentrations in diabetic patients with normoalbuminuria, microalbuminuria, and macroalbuminuria shown in Figs. 1 and 4. 


\begin{tabular}{|l|l|l|c|}
\hline All subjects & Non-diabetic subjects $(\mathbf{n}=\mathbf{9 7})$ & Patients with diabetes $(\mathbf{n}=\mathbf{7 7})$ & $\boldsymbol{p}$ \\
\hline Gender $($ Male/female $)$ & $64 / 33$ & $38 / 39$ & \\
\hline Age $($ years $)$ & $42(34,49)$ & $62(52,69)$ & $<0.001$ \\
\hline BMI $\left(\mathrm{kg} / \mathrm{m}^{2}\right)$ & $24(21,26)$ & $24(22,28)$ & 0.098 \\
\hline Type of diabetes $(1 / 2)$ & N/A & $31 / 46$ & \\
\hline Duration of diabetes (years) & N/A & $12(7.5,18)$ & \\
\hline HbAlc $(\%)$ & $5.4(5.2,5.6)$ & $7.1(6.7,7.7)$ & $<0.001$ \\
\hline eGFR $\left(\mathrm{mL} / \mathrm{min} / 1.73 \mathrm{~m}^{2}\right)$ & $88(76,96)$ & $68(55,83)$ & $<0.001$ \\
\hline Urinary albumin $(\mathrm{mg} / \mathrm{gCr})$ & $2.3(1.5,3.5)$ & $4.2(2.1,35)$ & $<0.001$ \\
\hline T-AN $(\mu \mathrm{g} / \mathrm{gCr})$ & $0.89(0.63,1.2)$ & $3.8(1.4,9.6)$ & $<0.001$ \\
\hline H-AN $(\mu \mathrm{g} / \mathrm{gCr})$ & $0.040(0.020,0.063)$ & $0.21(0.060,1.5)$ & $<0.001$ \\
\hline 42-64 years old only & Non-diabetic subjects $(\mathbf{n}=49)$ & Patients with diabetes $(\mathbf{n}=41)$ & $\boldsymbol{p}$ \\
\hline Gender $(\mathrm{Male} / \mathrm{Female})$ & $32 / 17$ & $20 / 21$ & \\
\hline Age $(\mathrm{years})$ & $49(44,56)$ & $54(46,59)$ & 0.083 \\
\hline BMI $\left(\mathrm{kg} / \mathrm{m}^{2}\right)$ & $24(21,27)$ & $25(22,29)$ & 0.38 \\
\hline Type of diabetes $(1 / 2)$ & N/A & $20 / 21$ & \\
\hline Duration of diabetes $(\mathrm{years})$ & N/A & $9(5.0,16)$ & \\
\hline HbAlc $(\%)$ & $5.5(5.2,5.8)$ & $7.2(6.8,8.1)$ & $<0.001$ \\
\hline eGFR $\left(\mathrm{mL} / \mathrm{min} / 1.73 \mathrm{~m}{ }^{2}\right)$ & $79(69,90)$ & $77(60,88)$ & 0.11 \\
\hline Urinary albumin $(\mathrm{mg} / \mathrm{gCr})$ & $2.4(1.7,3.9)$ & $3.3(1.9,36)$ & 0.011 \\
\hline T-AN $(\mu \mathrm{gg} / \mathrm{gCr})$ & $1.1(0.66,1.8)$ & $2.5(1.2,6.5)$ & $<0.001$ \\
\hline H-AN $(\mu \mathrm{gg} / \mathrm{gCr})$ & $0.043(0.020,0.074)$ & $0.11(0.051,0.57)$ & \\
\hline & Median $(25 \%, 75 \%)$ & & \\
\hline
\end{tabular}

Table 2. Characteristics of non-diabetic and diabetic groups. For comparison of differences between two groups, the Mann-Whitney's U test was used.

Relationship of T-AN and H-AN levels with DKD severity. Urine samples from 77 patients with either type 1 or type 2 diabetes and 97 non-diabetic controls were tested for T-AN, H-AN, albumin and creatinine. Characteristics of these two groups are summarized in Table 2. In this study, the non-diabetic subject group was a younger population (42, 34-49; Median, 25th-75th percentiles) than the diabetic patient group (62, 52-69), which might affect the levels of urinary biomarkers. Thus, a subgroup of 42-64 years old only was also investigated. The age of inclusion criteria in the analysis of 42-64 years was determined by the maximum age of non-diabetic subjects and the minimum age at which no statistical difference of age between the groups was observed.

Spearman's rank correlation coefficients between conventional biomarkers and T-AN or H-AN, all of which were normalized by creatinine, using all subjects' data are summarized in Table 3. T-AN and H-AN levels were strongly correlated with the albumin level in subjects with/without diabetes. In addition, the correlation between the T-AN level and eGFR and between the H-AN level and eGFR were stronger than that of the albumin level and eGFR especially in patients with diabetes. The same trends were observed in the age-restricted subgroup of 42-64 years old only (Table S2).

Levels of urinary biomarkers and eGFR at each albumin stage for all subjects are shown in Fig. 5a. Both T-AN and H-AN levels increased and eGFR decreased as the albumin stage progressed. Compared to nondiabetic subjects, T-AN and H-AN levels were increased significantly in diabetic patients of stage A1, A2, and A3. Similarly, T-AN and H-AN were elevated in stage A2 and A3 compared to stage A1. And further, T-AN and $\mathrm{H}-\mathrm{AN}$ were elevated in A3 compared to A2. The level of eGFR was also significantly decreased in stages A1, A2, and A3 compared with non-diabetic controls and a significant decrease was confirmed at stage A3, but not A2, compared to A1.

In the analysis of the age-restricted subgroup of 42-64 years old only (Fig. 5b), similar increases in T-AN and $\mathrm{H}-\mathrm{AN}$ levels were observed over non-diabetic subjects, increasing from A1 to A2 to A3 stages. Elevations were significant in all albumin stages over non-diabetic subjects. Further, H-AN levels were significantly elevated in stages A2, A3 over A1. But for T-AN, only stage A3 was significantly elevated over stage A1. For eGFR, only stage A3 showed a significant decrease from non-diabetic subjects or albumin stage A1 subjects.

Similar data analysis was performed based on eGFR stage in all subjects (Fig. 6a). Both T-AN and H-AN levels increased significantly as the eGFR stage progressed compared to non-diabetic subjects, with the exception of the H-AN levels of the G1 group which were not elevated. Albumin levels were increased significantly at eGFR stages G2, G3b, and G4 over non-diabetic subjects. Using G1 stage as baseline, no significant increase in T-AN was observed in progressive eGFR stages since the level of T-AN was already significantly high at the G1 stage. For H-AN, stage G3b levels were significantly elevated over G1 levels and this was observed also for albumin.

In the analysis of the age-restricted subgroup of 42-64 years old only (Fig. 6b), albumin and H-AN levels were statistically significantly increased, compared with non-diabetic subjects, at stage G3b whereas T-AN was statistically significantly increased in stages G2 and G3b. Similar to the results from all participants, T-AN and 


\begin{tabular}{|l|l|l|l|}
\hline \multicolumn{2}{|l|}{ ND } & DM & ND + DM \\
\hline \multicolumn{2}{|l|}{ Albumin } \\
\hline T-AN \\
\hline r & 0.25 & 0.70 & 0.54 \\
\hline p & 0.014 & $<0.001$ & $<0.001$ \\
\hline H-AN \\
\hline r & 0.47 & 0.75 & 0.66 \\
\hline p & $<0.001$ & $<0.001$ & $<0.001$ \\
\hline eGFR \\
\hline Albumin \\
\hline r & 0.013 & -0.32 & -0.23 \\
\hline$p$ & 0.90 & 0.0048 & 0.0025 \\
\hline T-AN & \multicolumn{5}{|l}{} \\
\hline r & -0.079 & -0.39 & -0.43 \\
\hline$p$ & 0.44 & $<0.001$ & $<0.001$ \\
\hline H-AN & & \\
\hline$r$ & 0.0017 & -0.49 & -0.38 \\
\hline$p$ & 0.99 & $<0.001$ & $<0.001$ \\
\hline
\end{tabular}

Table 3. Correlation between conventional biomarkers and T-AN or H-AN in the urine. ND, Non-diabetic subjects; DM, Type 1 and type 2 diabetes patients. Spearman's correlation coefficients were calculated.

H-AN levels in the stage G3a and T-AN, H-AN and albumin levels in the stage G4 were high, but the small number of patients prevents statistical significance to be achieved.

Whereas T-AN and H-AN levels were strongly associated with albumin stage, higher T-AN levels were observed at earlier stages of eGFR than was albumin. These results indicate that T-AN and H-AN may be useful as early biomarkers for DKD, although the response of T-AN and $\mathrm{H}-\mathrm{AN}$ levels to DKD severity is slightly different.

\section{Discussion}

We developed assays for measuring the amount of two adiponectin isoforms; one for T-AN levels and another for H-AN levels. To measure T-AN levels, two different monoclonal antibodies, both of which recognize the globular domain of adiponectin, were used to detect all types of adiponectin. In another assay, clone 38 antibody, which detects only HMW and MMW adiponectin (Fig.S1), was used as both capture and detection to react only with HMW, and weakly with MMW adiponectin. One of the strengths of our T-AN and H-AN assays is that they are fully automated on an immunoassay system, HI-1000. As a result, the measurement time was shortened from three days for the conventional manual ICT-EIA to approximately one hour. Our fully automated T-AN and $\mathrm{H}-\mathrm{AN}$ assays also show high reproducibility, good dilution linearity, and ultra-high sensitivity. In fact, the LoDs were 0.05 and $0.11 \mathrm{pg} / \mathrm{mL}$ for T-AN and H-AN assays, respectively, which would enable accurate measurement of very low levels of adiponectin in the urine of non-diabetic subjects (Fig. 5). Previous studies on urinary adiponectin used multiple commercially available Enzyme-linked immunosorbent assay kits ${ }^{4,7,8,10}$. These assays may differ in their reactivity to multiple isoforms of adiponectin, and reactivities of these assays to urinary adiponectin isoforms are still unknown. In this study, we developed two highly reproducible and sensitive assays that are fully automated, and have been characterized in detail with respect to the reactivity of these assays to all urinary adiponectin isoforms using SEC fractions of urine samples (Figs. 1 and 4).

Previous studies conducted by western blotting analysis have identified monomers, LMW and MMW adiponectin in urine $e^{6,13}$. Our ultrasensitive manual ICT-EIA could identify four types of adiponectin (HMW, MMW, LMW and monomer) by measurement of urine samples after SEC $^{29}$. As described above, the fully automated and newly developed T-AN and H-AN assays are also highly sensitive enough to measure extremely low concentrations of each adiponectin isoform in urine samples after SEC (Figs. 1 and 4). Thus, it was possible to compare the level of each adiponectin isoform in urine between three patients with diabetes, who have different stages of albuminuria, by using SEC samples of urine from them. Consistent with previous reports ${ }^{29}$, four types of adiponectin (HMW, MMW, LMW and monomer) were detected. Moreover, the results suggested that most urinary adiponectin in patients with normoalbuminuria are monomer and LMW isoform, whereas the proportion of macromolecules, such as HMW and MMW adiponectin, are higher in patients with albuminuria (Table 1 and Fig. 4). Several mechanisms of adiponectin excretion into the urine have been proposed in the past $^{8-10,12,13}$. Detection of more macromolecular adiponectin in patients with albuminuria supports a possibility that disruption of the glomerular molecular barrier may substantially contribute to an increased urinary adiponectin excretion in DKD.

Furthermore, we also showed that T-AN and H-AN levels were moderately to highly correlated with UAER and eGFR, which indicates that they may be used as biomarkers to tell the progression of DKD (Table 3). In fact, when T-AN and H-AN levels were measured in non-diabetic subjects and diabetic patients with various levels of $\mathrm{DKD}, \mathrm{T}$-AN level in diabetics became significantly higher than that in non-diabetic subjects from as early as the G1 stage (Fig. 6). A similar trend was observed with the age-restricted subgroup. H-AN levels showed a 
a) All subjects
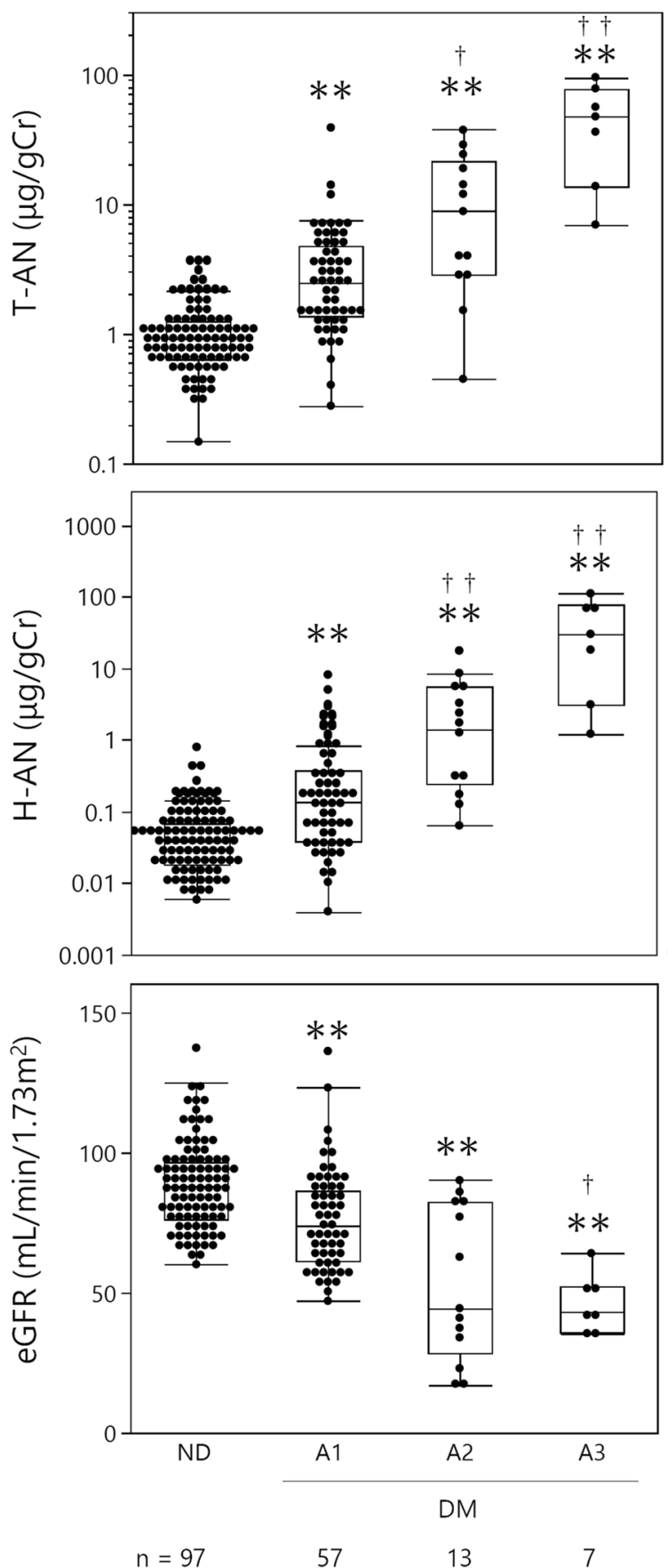

b) 42-64 years old only
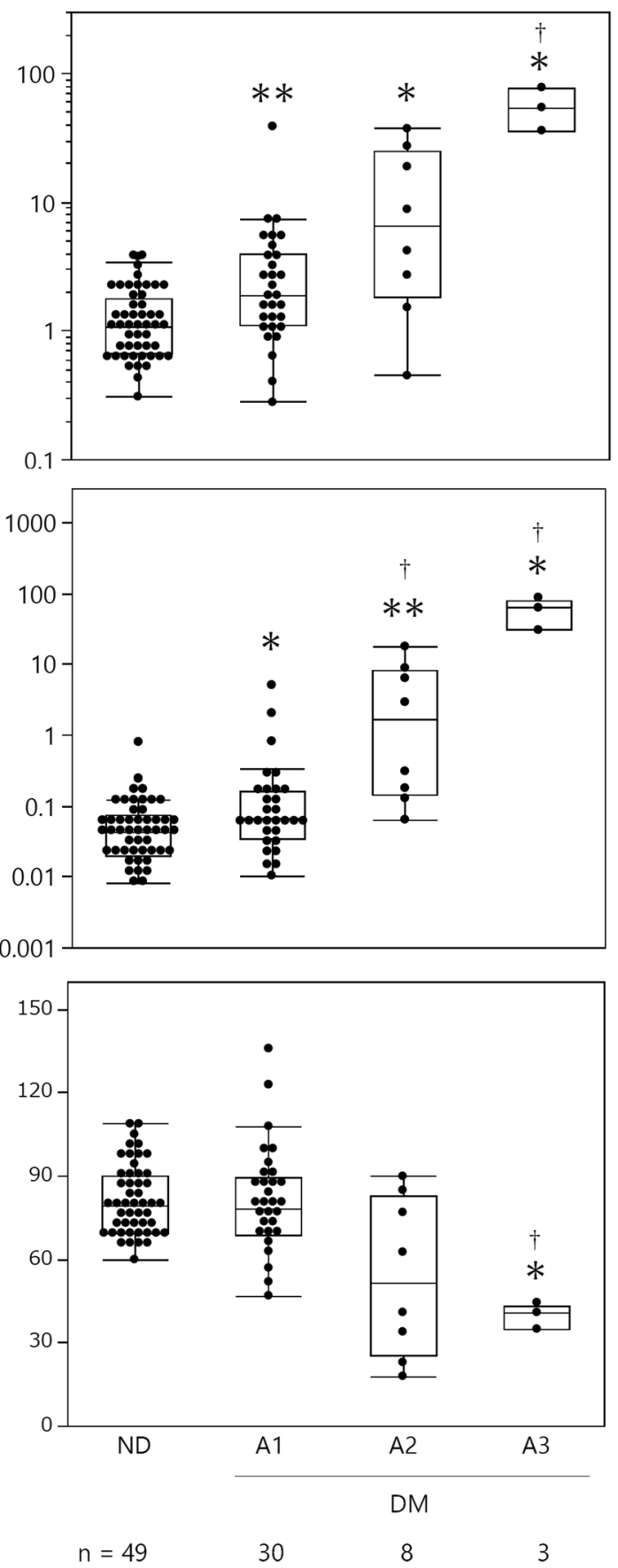

Figure 5. Levels of urinary biomarkers and eGFR at each albumin stage for all subjects (a) and subjects aged 42-64 years old only (b). Albumin stages were classified according to the following definition. A1: $<30$, A2: 30-299, A3: $\geqq 300 \mathrm{mg} / \mathrm{gCr}$. Kruskal-Wallis test and Steel-Dwass test were used for comparisons. ND: Nondiabetic subjects, DM: patients with Type 1 or type 2 diabetes, ${ }^{\star} P<0.05$ and ${ }^{*} P<0.01$ compared with ND, ${ }^{\dagger} P<0.05$ and ${ }^{\dagger \dagger} P<0.01$ compared with A1.

similar trend to albumin, with elevations observed in diabetics with > G2 stage when compared with those in non-diabetics. Thus, T-AN and H-AN levels may serve as biomarkers for DKD: usage of T-AN assay to detect a wide variety of adiponectin isoforms may enable detection of early pathological changes in the kidney. On the other hand, H-AN levels could be a biomarker of more progressed DKD.

In conclusion, we developed two ICT-EIAs for measuring T-AN and H-AN which showed high reproducibility, good dilution linearity, and ultra-high sensitivity. The conventional ICT-EIA method took 3 days to test samples, but the newly developed assays are fully automated and can finish an assay in $1 \mathrm{~h}$ by using a 
a) All subjects

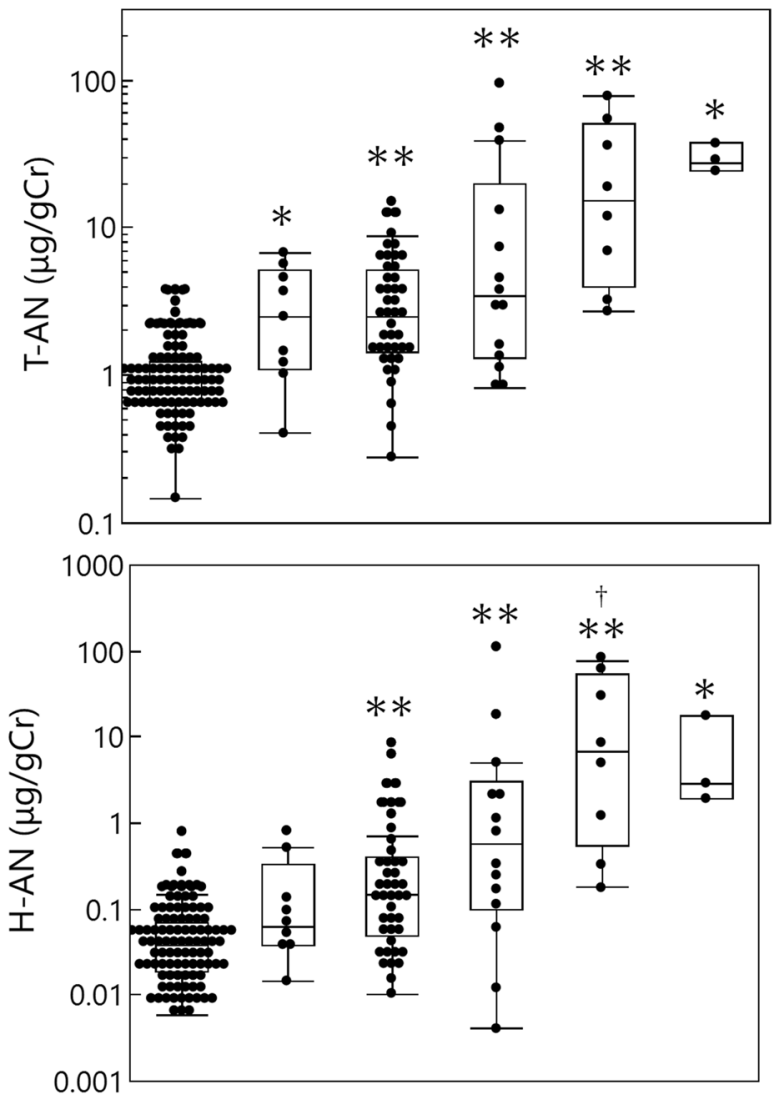

b) 42-64 years old only
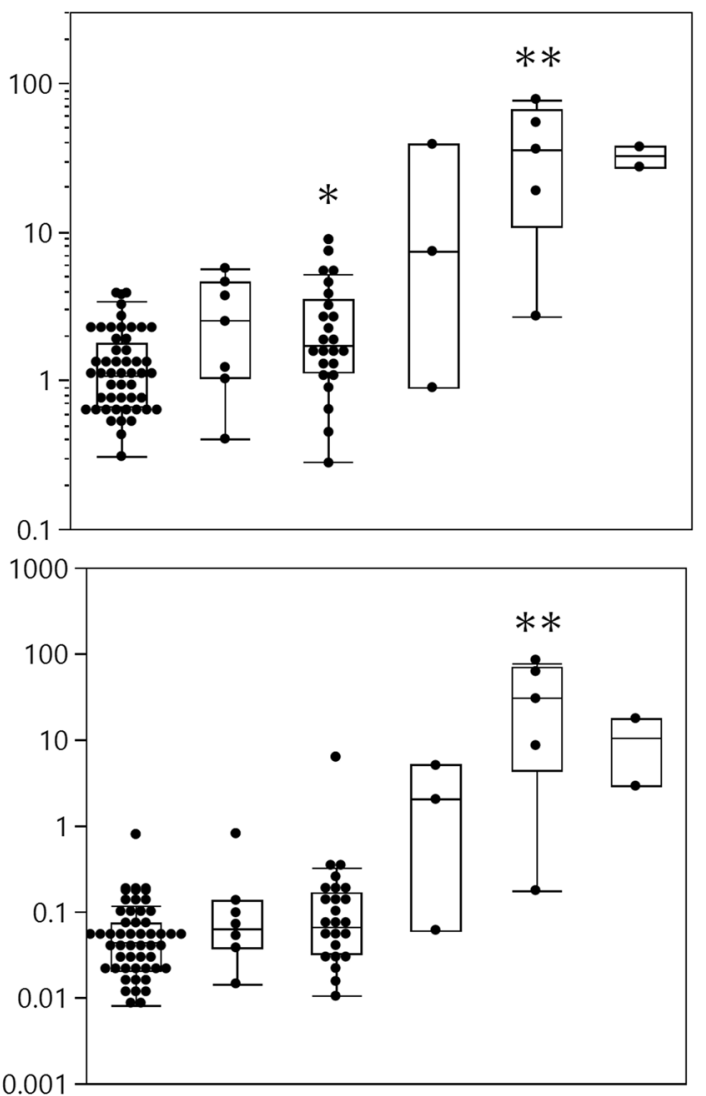
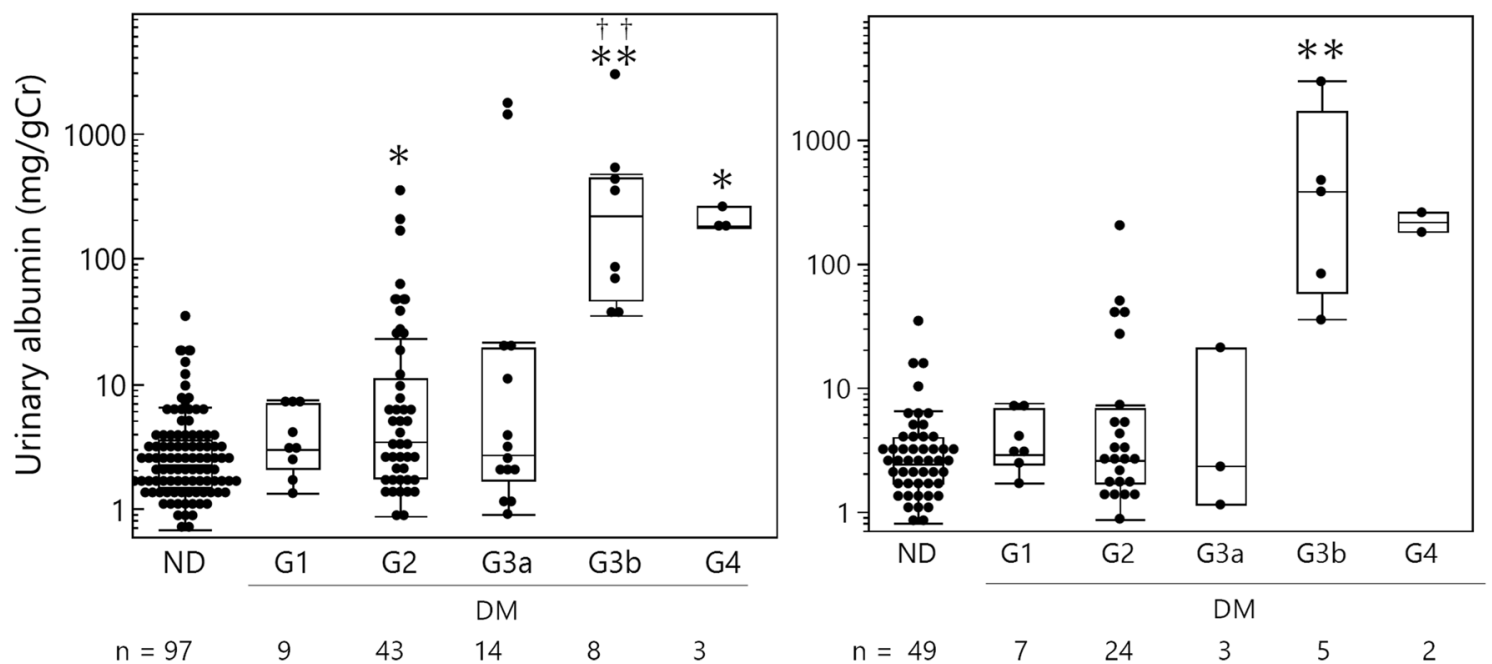

Figure 6. Levels of urinary biomarkers at each eGFR stage for all subjects (a) and subjects aged 42-64 years old only (b). eGFR stages were classified according to the following definition. G1: $\geqq 90$, G2: 60-89, G3a: 45-59, G3b: 30-44, G4: 15-29 ml/min/1.73 $\mathrm{m}^{2}$. Kruskal-Wallis test and Steel-Dwass test were used for comparisons. ND: Non-diabetic subjects, DM: patients with Type 1 or type 2 diabetes, ${ }^{\star} P<0.05$ and ${ }^{* \star} P<0.01$ compared with ND, ${ }^{\dagger} P<0.05$ and ${ }^{\dagger \dagger} P<0.01$ compared with G1.

high-sensitivity immunoassay system, the HI-1000. Furthermore, using these new assays, we showed that both $\mathrm{T}-\mathrm{AN}$ and $\mathrm{H}$-AN levels in the urine may be increased in patients with diabetes and they may have clinical significance in tackling DKD.

The limitations of this study are limited clinical data, cross-sectional studies, age-biased controls, and lack of accurate diagnosis of diabetic renal disease, which could be made by a biopsy. In the future, the usefulness of urinary adiponectin levels as a biomarker of DKD should be tested in larger prospective studies, which is currently underway. 


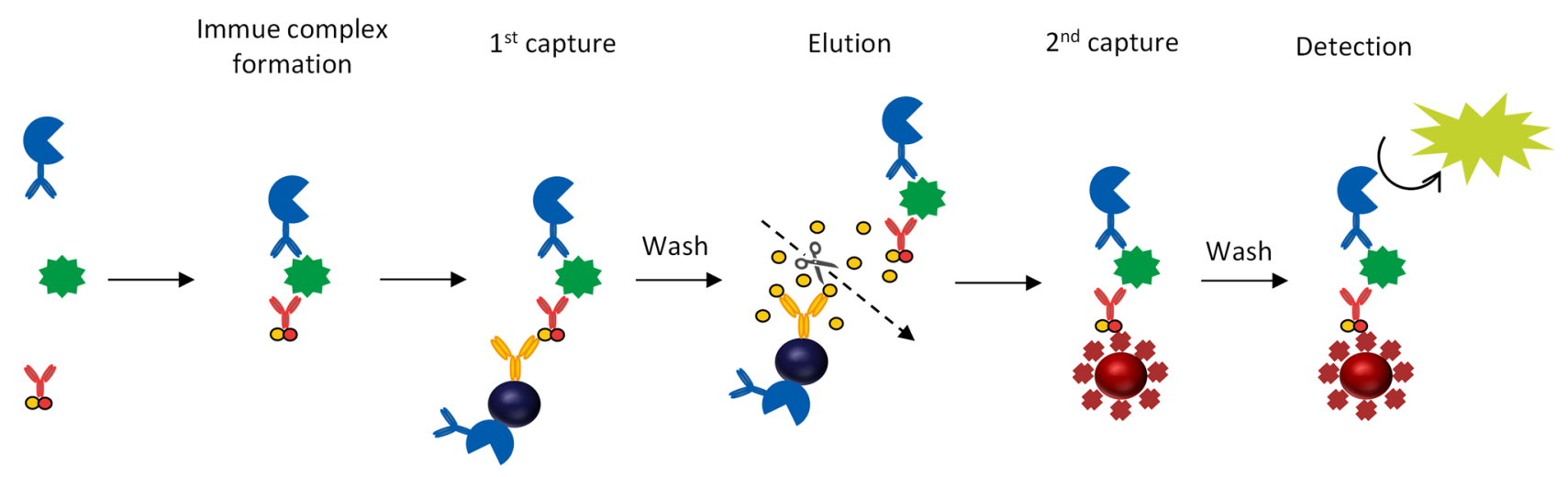

Figure 7. Assay scheme of ICT-EIA. All reactions were performed on HI-1000. Immune complexes formed in the liquid phase are captured by anti-DNP antibody beads. After washing, the immune complex is released into the liquid phase by the addition of large amounts of DNP-Lys and re-captured by streptavidin beads. After a second wash, the amount of chemiluminescence produced by the enzyme-substrate reaction is measured ${ }^{24}$.

\begin{abstract}
Methods
Urinary adiponectin assay. Buffers. $10 \mathrm{mM}$ sodium phosphate buffer ( $\mathrm{pH} 7.0$ ) containing $0.1 \mathrm{M}$ sodium chloride $(\mathrm{NaCl}), 1.0 \mathrm{mM}$ magnesium chloride $\left(\mathrm{MgCl}_{2}\right), 0.1 \%$ sodium azide $\left(\mathrm{NaN}_{3}\right)$ and $0.1 \%$ bovine serum albumin (BSA) was used for dialysis and dilution of urine samples. 0.1 M 2-(N-morpholino)ethanesulfonic acid (MES) buffer ( $\mathrm{pH}$ 6.5) containing $0.15 \mathrm{M} \mathrm{NaCl}, 1.0 \mathrm{mM} \mathrm{MgCl}, 0.1 \mathrm{mM}$ zinc chloride $\left(\mathrm{ZnCl}_{2}\right), 0.1 \% \mathrm{NaN}_{3}$ and $0.1 \%$ BSA was used for dilution of detection antibody conjugates. The same composition without $\mathrm{MgCl}_{2}$ and $\mathrm{ZnCl}_{2}$ was used for dilution buffer for capture antibody conjugates. $0.1 \mathrm{M}$ MES buffer ( $\mathrm{pH}$ 6.5) containing $2.5 \mathrm{mM} \mathrm{N \varepsilon}$-DNP-L-lysine hydrochloride (DNP), $2 \%$ casein sodium and $0.1 \% \mathrm{NaN}_{3}$ was used for elusion buffer in ICT-EIA. HISCL washing solution (Sysmex, Hyogo, Japan) was used for washing buffer.
\end{abstract}

Antibodies and antigens. Different types of antibodies were employed to measure T-AN levels and H-AN levels, respectively. Monoclonal mouse anti-human Adiponectin/Acrp30 antibody (Product code: MAB10651, Clone: 166126, Antibody Registry: AB_2221612) and monoclonal mouse anti-human Adiponectin/Acrp30 antibody (Product code: MAB1065, Clone: 166128, Antibody Registry: AB_2273512) were chosen as capture and detection antibodies, respectively, for T-AN assay. Monoclonal mouse anti-human Adiponectin/Acrp30 antibody (Clone: 38, Sysmex, Hyogo, Japan) was used for both capture and detection antibodies for H-AN assay. Recombinant Human Adiponectin (Oriental yeast, Tokyo, Japan) was used as calibrators.

Preparation of capture and detection antibody conjugates. For T-AN assays, thiol groups were introduced into the capture antibody (Clone: 166126) using PIERCE SATA (N-succinimidyl S-acetylthioacetate) (Thermo Fisher Scientific Inc., Waltham, MA) and the antibody was then conjugated with 6-maleimidohexanoyl-DNP and 6-maleimidohexanoyl-biocytin ${ }^{30,31}$. Other monoclonal antibodies were digested with pepsin (Roche, Basel, Switz) to F(ab')2, which was further reduced to obtain Fab'. Fab' fragments were conjugated with 6-maleimidohexanoyl-DNP-biotinyl-BSA as capture antibody conjugate and with alkaline phosphatase from calf intestine (Oriental yeast, Tokyo, Japan) using N-( -maleimidocaproyloxy)succinimide (Dojindo, Kumamoto, Japan) as detection antibody conjugate ${ }^{30,31}$.

Preparation of protein-coated magnetic particles. Carboxyl groups on their surface of magnetic particles (JSR, Tokyo, Japan) were activated by using 1-Ethyl-3-(3-dimethylaminopropyl)carbodiimide, hydrochloride (Dojindo, Kumamoto, Japan) and N-Hydroxysuccinimide (Tokyo Chemistry Industry, Tokyo, Japan). The activated magnetic particles were mixed and coated with monoclonal mouse anti-DNP antibody (Clone: 1853, Sysmex, Hyogo, Japan) and Biotinyl-BSA, respectively.. Biotinyl-BSA-coated magnetic particles were then reacted

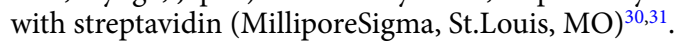

Immune complex transfer enzyme immunoassay (ICT-EIA) for adiponectin. The protocol of the ICT-EIA for T-AN was as follows (Fig. 7): $70 \mu \mathrm{L}$ of sample was mixed with $40 \mu \mathrm{L}$ of detection antibody conjugate and incubated for $3.4 \mathrm{~min} .40 \mu \mathrm{L}$ of capture antibody conjugate was added and incubated for $23.8 \mathrm{~min}$. After adding $20 \mu \mathrm{L}$ of anti-DNP antibody coated magnetic particles, immune complexes were captured onto the surface of the beads during 11.3 min of incubation. After washing, the immune complexes were released from the beads surface during $4.6 \mathrm{~min}$ of incubation in $110 \mu \mathrm{L}$ of elution buffer. With magnetic particles magnetized, $80 \mu \mathrm{L}$ of the supernatant of the reaction solution containing the immune complexes was taken and transferred to another vial. After adding $30 \mu \mathrm{L}$ of streptavidin coated magnetic particles, immune complexes were re-captured onto the surface of the beads during $5.3 \mathrm{~min}$ of incubation. All reactions up to this point were performed at $37^{\circ} \mathrm{C}$. The bead was then washed, and the bound alkaline phosphatase activity was assayed by chemiluminescence with HISCL substrate reagent set (Sysmex, Hyogo, Japan) for 5.0 min at $42^{\circ} \mathrm{C}$. H-AN was measured in a similar manner, except that the reaction time with the detection antibody conjugate was $5.8 \mathrm{~min}$ and with the capture 
antibody conjugate was $9.4 \mathrm{~min}$. All reactions were performed automatically using a high-sensitivity immunoassay system, the HI-1000 (Sysmex, Hyogo, Japan).

Assay characterization. Calibration curves were generated with 9 calibrators spanning a dynamic range of 0 to $1000 \mathrm{pg} / \mathrm{mL}$ for T-AN assay and 6 calibrators spanning a dynamic range of 0 to 10,000 pg/mL for H-AN assay. The LoD was calculated as the concentration which is equivalent to the mean plus 3 standard deviations of low concentration samples. The calibrators were measured 10 times to determine reproducibility, and coefficients of variations were calculated. In a dilution linearity evaluation, a dialyzed urine sample was diluted with dilution buffer to achieve 4 new concentrations. These 4 dilutions and the original calibrator were measured 3 times, respectively, and the difference between the concentration of the original sample and the expected concentrations calculated from the concentration of the diluted samples and their dilution factors were compared.

\section{Clinical study}

Subjects and samples. Nighty seven non-diabetic subjects from an occupational-based cohort study held in Tokushima prefecture and 77 outpatients with type 1 or type 2 diabetes in Tokushima University Hospital were enrolled in this study. Non-diabetic subjects were defined as satisfying the following three points: fasting blood glucose level was less than $126 \mathrm{mg} / \mathrm{dl}$, HbAlc was less than 6.5\%, and no diabetes medication was prescribed. Diabetes was defined by physicians according to the diagnostic criteria of Japan Diabetes Society ${ }^{32}$. Blood samples were drawn from an antecubital vein of subjects. Early morning urine samples were collected after overnight fasting. The urine samples $(10 \mathrm{~mL})$ were mixed with $0.1 \mathrm{~mL}$ of $10 \% \mathrm{BSA}$ and $\mathrm{NaN}_{3}$ and dialyzed against dialysis buffer at $4{ }^{\circ} \mathrm{C}$ overnight. Dialyzed urine samples were kept frozen at $-30{ }^{\circ} \mathrm{C}$ until analysis.

Size exclusion chromatography (SEC) of urine. Three randomly chosen urine samples (1.0 mL) from patients with normoalbuminuria, microalbuminuria and macroalbuminuria, respectively, were selected from patients with diabetes and separated by SEC using an AKTA explorer 10S (GE Healthcare, Tokyo, Japan) on a column of HiLoad 16/60 Superdex 200 prep grade $(1.6 \times 60 \mathrm{~cm})$ (GE Healthcare). An aliquot of each fraction was used for T-AN and H-AN assays.

Measurements of biomarkers in urine. Dialyzed urine samples were analyzed for adiponectin, using T-AN and H-AN as described above. They were also analyzed for albumin, by an ICT-EIA for albumin ${ }^{29}$ and creatinine by a commercially available creatinine kit (Creatinine-test Wako, FUJIFILM Wako Pure Chemical Corporation, Osaka, Japan). Urinary adiponectin and albumin concentrations were normalized by the urinary creatinine $(\mathrm{Cr})$ concentration. Urine samples after SEC fractionation were also tested for T-AN and H-AN.

Statistical analysis. The Shapiro-Wilk test was used to examine whether the measurements of biomarkers were normally distributed. For biomarker correlation analysis, Spearman's correlation coefficients were calculated. For comparison of differences between two or multiple groups, the Mann-Whitney's U test or the Kruskal-Wallis test and Steel-Dwass test were used, with $5 \%$ as the criterion for significance.

Ethical considerations. The study protocol was approved by the ethical committee of Tokushima Bunri University (No. H29-17) and by the ethical committee of Tokushima University Hospital (No. 2894-1 for diabetics and No. 3087 for non-diabetics); all diabetic participants gave written informed consent. Non-diabetic participants were participants of an occupational-based cohort study, which was also approved by the ethical committee of Tokushima University Hospital (No. 662), and gave written informed consent to this cohort study. In No. 3087, non-diabetic participants were provided an easy mode to opt-out by a hand-out and online so that participants are well-informed about this study and their opportunity to indicate refusal. All methods were performed in accordance with the relevant guidelines and regulations.

\section{Data availability}

The datasets generated during and/or analyzed during the current study are available from the corresponding author on reasonable request.

Received: 23 July 2020; Accepted: 31 August 2020

Published online: 28 September 2020

\section{References}

1. Levey, A. S. et al. Definition and classification of chronic kidney disease: a position statement from Kidney Disease: Improving Global Outcomes (KDIGO). Kidney Int. 67, 2089-2100 (2005).

2. Sarnak Mark, J. et al. Kidney disease as a risk factor for development of cardiovascular disease. Circulation 108, 2154-2169 (2003).

3. National Kidney Foundation. K/DOQI clinical practice guidelines for chronic kidney disease: evaluation, classification, and stratification. Am. J. Kidney Dis. 39, S1-266 (2002).

4. Koshimura, J. et al. Urinary adiponectin excretion is increased in patients with overt diabetic nephropathy. Biochem. Biophys. Res. Commun. 316, 165-169 (2004).

5. Jorsal, A. et al. Urinary adiponectin excretion rises with increasing albuminuria in type 1 diabetes. J. Diabetes Compl. 27, 604-608 (2013).

6. Yamakado, S. et al. Urinary adiponectin as a new diagnostic index for chronic kidney disease due to diabetic nephropathy. BMJ Open Diabetes Res. Care 7, e000661 (2019).

7. Kopf, S. et al. Urinary excretion of high molecular weight adiponectin is an independent predictor of decline of renal function in type 2 diabetes. Acta Diabetol. 51, 479-489 (2014). 
8. Panduru, N. M. et al. Urinary adiponectin is an independent predictor of progression to end-stage renal disease in patients with type 1 diabetes and diabetic nephropathy. Diabetes Care 38, 883-890 (2015).

9. Shimotomai, T. et al. Enhanced urinary adiponectin excretion in IgA-nephropathy patients with proteinuria. Ren. Fail. 27, 323-328 (2005).

10. Rovin, B. H. et al. Plasma, urine, and renal expression of adiponectin in human systemic lupus erythematosus. Kidney Int. 68, 1825-1833 (2005).

11. Loghman, M. et al. Association between urinary adiponectin level and renal involvement in systemic lupus erythematous. Int. J. Rheum. Dis. 19, 678-684 (2016).

12. Fujita, H. et al. Possible relationship between adiponectin and renal tubular injury in diabetic nephropathy. Endocr. J. 53, 745-752 (2006).

13. von Eynatten, M. et al. Urinary adiponectin excretion: a novel marker for vascular damage in type 2 diabetes. Diabetes 58, 2093-2099 (2009).

14. Huang, L., Chen, J., Yu, Z. \& Tang, D. Self-powered temperature sensor with seebeck effect transduction for photothermalthermoelectric coupled immunoassay. Anal. Chem. 92, 2809-2814 (2020).

15. Yu, Z., Tang, Y., Cai, G., Ren, R. \& Tang, D. Paper electrode-based flexible pressure sensor for point-of-care immunoassay with digital multimeter. Anal. Chem. 91, 1222-1226 (2019).

16. Luo, Z. et al. Branched polyethylenimine-modified upconversion nanohybrid-mediated photoelectrochemical immunoassay with synergistic effect of dual-purpose copper ions. Anal. Chem. 91, 4149-4156 (2019).

17. Akama, K. et al. Wash- and amplification-free digital immunoassay based on single-particle motion analysis. ACS Nano 13, 13116-13126 (2019).

18. Akama, K. \& Noji, H. Multiplexed homogeneous digital immunoassay based on single-particle motion analysis. Lab Chip 20, 2113-2121 (2020).

19. Eom, S. et al. Development of recombinant immunoglobulin G-binding luciferase-based signal amplifiers in immunoassays. Anal. Chem. 92, 5473-5481 (2020).

20. Tobos, C. I., Sheehan, A. J., Duffy, D. C. \& Rissin, D. M. Customizable multiplex antibody array immunoassays with attomolar sensitivities. Anal. Chem. 92, 5613-5619 (2020).

21. Byrnes, S. A. et al. Wash-free, digital immunoassay in polydisperse droplets. Anal. Chem. 92, 3535-3543 (2020).

22. Sampedro, F. et al. Increased plasma neurofilament light chain levels in patients with type-1 diabetes with impaired awareness of hypoglycemia. BMJ Open Diabetes Res Care 8, e001516 (2020).

23. Kaess, B. M. et al. Ultra-sensitive troponin I is an independent predictor of incident coronary heart disease in the general population. Eur. J. Epidemiol. 32, 583-591 (2017).

24. Watanabe, T. \& Hashida, S. The immune complex transfer enzyme immunoassay: Mechanism of improved sensitivity compared with conventional sandwich enzyme immunoassay. J. Immunol. Methods 459, 76-80 (2018).

25. Chevaliez, S. et al. Multicenter clinical evaluation of alinity m HCV assay performance. J. Clin. Virol. 129, 104531 (2020).

26. Georgakopoulou, V. E. et al. Correlation between serum levels of 25-hydroxyvitamin D and severity of community-acquired pneumonia in hospitalized patients assessed by Pneumonia Severity Index: An Observational Descriptive Study. Cureus 12, e8947 (2020).

27. Agnello, L. et al. Diagnostic accuracy of cerebrospinal fluid biomarkers measured by chemiluminescent enzyme immunoassay for Alzheimer disease diagnosis. Scand. J. Clin. Lab. Invest. 80, 313-317 (2020).

28. Jekarl, D. W. et al. Analytical and clinical evaluation of chemiluminescent carcinoembryonic antigen (CEA) by HISCL-5000 Immunoanalyzer. Ann. Clin. Lab. Sci. 50, 417-422 (2020).

29. Yamamoto, M., Fujimoto, Y., Hayashi, S. \& Hashida, S. A study of high-, middle- and low-molecular weight adiponectin in urine as a surrogate marker for early diabetic nephropathy using ultrasensitive immune complex transfer enzyme immunoassay. Ann. Clin. Biochem. 55, 525-534 (2018).

30. Hashida, S. \& Ishikawa, E. Detection of one milliattomole of ferritin by novel and ultrasensitive enzyme immunoassay. J. Biochem. 108, 960-964 (1990).

31. Hashida, S., Hashinaka, K. \& Ishikawa, E. Ultrasensitive enzyme immunoassay. In Biotechnology Annual Review (ed. El-Gewely, M. R.) Vol. 1 403-451 (Elsevier, New York, 1995).

32. The Committee of the Japan Diabetes Society on the Diagnostic Criteria of Diabetes Mellitus et al. Report of the Committee on the Classification and Diagnostic Criteria of Diabetes Mellitus. J. Diabetes Investig. 1, 212-228 (2010).

\section{Acknowledgements}

The authors thank the following laboratory researcher: Yumiko Hisabae. We also thank Tracey Colpitts for carefully proofreading the manuscript.

\section{Author contributions}

T.W. contributed to the study concept and design, follow-up, data research, statistical analyses, and writing of the manuscript. Y.F. contributed to the data collection and data research, statistical analyses, and discussion. A.M., M.N., A.K. and S.O. contributed to the study concept and design, follow-up, data research. M.A., T.K. and M.K. contributed to the data collection and data research. M.I., H.M., M.M., A.H. and M.F. contributed to the sample collection and discussion. SH contributed to the study concept and design, data research, statistical analyses, discussion. All authors reviewed and edited the manuscript and approved the final version of the manuscript.

\section{Competing interests}

MM, MF and SH received research funding from Sysmex Corporation. TW is an employee of Sysmex R\&D Center Americas, Inc. AM, MN, and SO are employees of Sysmex Corporation.

\section{Additional information}

Supplementary information is available for this paper at https://doi.org/10.1038/s41598-020-72494-6.

Correspondence and requests for materials should be addressed to T.W.

Reprints and permissions information is available at www.nature.com/reprints.

Publisher's note Springer Nature remains neutral with regard to jurisdictional claims in published maps and institutional affiliations. 
(c) (i) Open Access This article is licensed under a Creative Commons Attribution 4.0 International cc) License, which permits use, sharing, adaptation, distribution and reproduction in any medium or format, as long as you give appropriate credit to the original author(s) and the source, provide a link to the Creative Commons licence, and indicate if changes were made. The images or other third party material in this article are included in the article's Creative Commons licence, unless indicated otherwise in a credit line to the material. If material is not included in the article's Creative Commons licence and your intended use is not permitted by statutory regulation or exceeds the permitted use, you will need to obtain permission directly from the copyright holder. To view a copy of this licence, visit http://creativecommons.org/licenses/by/4.0/.

(C) The Author(s) 2020 